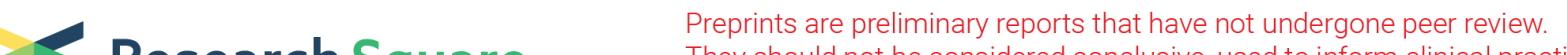 Research Square They should not be considered conclusive, used to inform clinical practice, or referenced by the media as validated information.
}

\section{A Novel Nomogram for Predicting Cancer-specific Survival in Women With Uterine Sarcoma: a Large Population-based Study}

\author{
Yuan-jie Li \\ school fo Basic medical Sciences, Xi'an Jiaotong University \\ Jun Lyu \\ the First Affiliated Hospital of Jinan University \\ Chen Li \\ the First Affiliated Hospital of Xi'an Jiaotong University \\ Hai-rong He \\ the First Affiliated Hospital of Xi'an Jiaotong University \\ Jin-feng Wang \\ the First Affiliated Hospital of Xi'an Jiaotong University \\ Yue-ling Wang \\ the First Affiliated Hospital of Xi'an Jiaotong University \\ Jing Fang \\ the First Affiliated Hospital of Xi'an Jiaotong University \\ Jing Ji ( $\nabla_{\text {jijing08@xjtu.edu.cn ) }}$ \\ the First Affiliated Hospital of Jinan University
}

\section{Research Article}

Keywords: Nomogram, Uterine sarcoma, SEER database, Cancer-specific survival

Posted Date: June 22nd, 2021

DOI: https://doi.org/10.21203/rs.3.rs-630236/v1

License: (c) (1) This work is licensed under a Creative Commons Attribution 4.0 International License. Read Full License 


\section{Abstract}

Background: To perform a comprehensive nomogram to predict the cancer-specific survival (CSS) for uterine sarcoma (US) based on the Surveillance, Epidemiology, and End Results (SEER) database.

Methods: A total of 3861 patients with US between 2010 and 2015 were identified in this study. They were randomly divided into a training cohort $(n=2702)$ and a validation cohort $(n=1159)$ in a 7-to-3 ratio by $\mathrm{R}$ software. We performed multivariate Cox analysis to select predictive variables and identify independent prognostic factors. Then, the discrimination and calibration of the nomogram were evaluated by concordance index (C-index) and the area under the curve (AUC). Finally, the net reclassification improvement (NRI), the integrated discrimination improvement (IDI), calibration plotting, and decision-curve analysis (DCA) were used to evaluate the benefits of the new prediction model.

Results: We have established a nomogram to predict 1-, 3-, and 5-year CSS for US patients. In this nomogram, pathology grade has the highest risk on CSS in US, followed by age at diagnosis, then surgery status. Comparing to the AJCC staging system, the nomogram showed better predictive discrimination with higher $\mathrm{C}$-index in both training and validation cohort ( 0.796 and 0.767 vs 0.706 and 0.713 , respectively). Furthermore, AUC value, calibration plotting, NRI, IDI, and DCA also demonstrated better performance than the traditional system.

Conclusion: Our study validated the first comprehensive nomogram for US which could provide more accurately and individualized survival predictions for US patients in clinical practice.

\section{Background}

Uterine Sarcoma (US) is a rare malignant uterine tumor in women that account for $3-7 \%$ of all uterine cancer cases [1] and is characterized by aggressive behavior and rapid progression. The incidence of US ranges from 1.55 to 1.95 per 100000 females per year [2]. Current classification of US includes endometrial stromal sarcoma, leiomyosarcoma, mixed epithelial and mesenchymal tumors according to the common histological types [3]. No common etiology has been identified, but several agents might be associated US, such as tamoxifen treatment, pelvic radiation therapy, and hereditary leiomyomatosis [4]. Management involves the coordination of multidisciplinary treatment including surgery, radiotherapy, chemotherapy and hormonal blockade. However, the 5 -year survival rate is less than $50 \%$ in early stages and less than $15 \%$ in advanced stages $[5,6]$.

The American Joint Committee on Cancer (AJCC) staging system is the most extensively used clinical tool in determining the prediction of cancer [7], which is based on the tumor $(T)$, number of metastatic lymph nodes $(\mathrm{N})$, and distant metastasis $(\mathrm{M})$. However, US is a very heterogeneous disease. Patients' response to therapy differs widely and the survival rate varies at the same stage. It was known that some of the clinical characteristics such as age, race, tumor size were also noteworthy factors influencing individual survival outcomes of cancer patients $[8,9]$. For example, US is twice more frequent among black women than that of white women, also, the risk of sarcoma is higher for women aged 50 years [10]. 
Thus, a novel exact prognostic tool which contains personalized characteristics is needed to improve the accuracy of prognosis in women with US.

Recently, nomogram which presented by grahphs is widely used to predict an outcome of malignant tumors. This study aimed to develop an effective nomogram to predict the cancer-specific survival (CSS) of US patients based on a cohort from the SEER database, and to explore the relative demographic factors and clinicopathological features.

\section{Methods}

\section{Data source}

All the related information were derived from the SEER program (the latest version which covering 18 registries additional chemotherapy data), by using SEER*Stat version 8.3.6.1 (https://seer.cancer.gov/) [11-13]. The population included in this study were the patients diagnosed as uterine sarcoma between 2010 to 2015 , with the ICD-0-3 morphology codes as follows [10]:8800/3-8805/3; 8890/3, 8891/3, $8896 / 3 ; 8933 / 3 ; 8930 / 3,8931 / 3,8935 / 3 ; 8950 / 3,8951 / 3,8980 / 3$.

We also chose demographic and clinicopathological features in this study, including race, age at diagnosis, marital status, tumor size, pathology grade, SEER stage, AJCC stage, surgery, chemotherapy, and radiotherapy. We noticed that the summary stage in the SEER database has four levels: in situ, localized, regional, and distant. In our study, we only included the last three levels due to the lack of patient data in the first one. Tumor pathology grade is divided into four levels according to the degree of differentiation: Grade I (well), II (moderately), III (poorly), and IV (undifferentiated or anaplastic) [12]. The tumor size was classified as $\leq 50 \mathrm{~mm},>50 \mathrm{~mm}$ and unknown. Based on the SEER database, surgery status was divided into two groups: "Yes" means surgery performed, while "No" means no surgery performed due to three situations as follow: not recommended, recommended but patient refused or died before surgery. Radiotherapy status was also classified. "Yes" means radiation preformed including radiation, radioactive implants, beam radiation, and combination of beam with implants or isotopes [12], while "No" means none/unknown, refused, or recommended but unknown if administered. Chemotherapy status was classified as: "Yes" means chemotherapy performed, and "No" means not performed or unknown. The endpoint of this study was death of patients due to US.

\section{Criteria for data selection}

The followings were exclusion criteria: 1) patients with no confirmation by microscopy or only in an autopsy; 2) no tumor found; 3) unknown insurance status. The retrospective study initially identified 3922 uterine sarcoma patients enrolled in the SEER database from 2010 to 2015, of which 3861 patients were finally included based on the criteria mentioned above (Fig. 1).

\section{Statistical analysis}


Twelve pathological and clinical features that we mentioned above were applied to conduct the analyses. Cox regression was developed to assess the effects of correlation factors $(p=0.1)$. Then the differentiation ability of the nomogram was evaluated using $\mathrm{C}$-index and the calibration curves. We made comparison of the accuracy and comprehensiveness of our nomogram with the AJCC stage by using NRI and IDI [14]. The consistency of survival probabilities predicted by the nomogram with the actual situation was assessed by calibration plots, and clinical validity of nomogram was tested via DCA [15].

All statistical analyses and graphics were performed by R software (version 3.6.0; http://www.Rproject.org) and SPSS software (version 24.0, SPSS, Chicago, IL, USA), with $P<0.05$ considered to have statistical significance.

\section{Results}

\section{Patients' characteristics}

3861 US patients extracted from the SEER database were randomly divided into training cohort $(n=2702)$ and a validation cohort $(n=1159)$ with a ratio of $7: 3$. The median age at diagnosis in the training and validation cohorts was 62 years (interquartile range, 53-69 years) and 60 years (interquartile range, 5169 years), respectively. The majority of the patients were white (69.2 and 71.2\%), married (47.6 and $48.3 \%$ ), and insured (95.4 and 96.3\%). Most of the tumors were at pathological Grade III or IV and bigger than $50 \mathrm{~mm}$ in both cohorts. Nearly half of the patients were histologically diagnosed with carcinosarcoma. The distribution of different SEER stages was close to agreement with a little higher rate in localized group (37.4 and 42.5\%). Nearly half of the patients were in AJCC stage I (41.0 and 45.1\%) and only less than one tenth were in AJCC stage II (9.7 and 8.1\%). Most of the patients received surgery (90.8 and $92.4 \%$ ), with a few receiving radiotherapy and over half receiving chemotherapy in both cohorts.

The summary of these clinical characteristics was listed in Table 1. 
Table 1

Patient characteristics in the study

\begin{tabular}{|c|c|c|}
\hline Variable & $\begin{array}{l}\text { Training Cohort } \\
(n=2702)\end{array}$ & $\begin{array}{l}\text { Validation Cohort } \\
(n=1159)\end{array}$ \\
\hline $\begin{array}{l}\text { Medium age at diagnosis, } \\
\text { (25th }-75 \text { th percentile) }\end{array}$ & $62(53-69)$ & $60(51-69)$ \\
\hline \multicolumn{3}{|l|}{ Race n (\%) } \\
\hline White & $1869(69.2)$ & $808(71.2)$ \\
\hline Black & $591(22.1)$ & $243(20.2)$ \\
\hline Other & $242(8.6)$ & $108(8.6)$ \\
\hline \multicolumn{3}{|l|}{ Marital status n (\%) } \\
\hline Married & $1257(47.6)$ & $561(48.3)$ \\
\hline Single & $586(22.3)$ & $261(22.7)$ \\
\hline SDW & $736(25.6)$ & $293(25.5)$ \\
\hline Unknown & $123(4.6)$ & $44(3.5)$ \\
\hline \multicolumn{3}{|l|}{ Insurance record n (\%) } \\
\hline Yes & $2584(95.4)$ & $1117(96.3)$ \\
\hline No & $118(4.6)$ & $42(3.7)$ \\
\hline \multicolumn{3}{|l|}{ Tumor size n (\%) } \\
\hline$\leq 50 \mathrm{~mm}$ & $710(23.1)$ & $313(23.8)$ \\
\hline$>50 \mathrm{~mm}$ & $1596(61.4)$ & $677(61.6)$ \\
\hline Unknown & $396(15.6)$ & $169(14.6)$ \\
\hline \multicolumn{3}{|l|}{ Pathological grade n (\%) } \\
\hline I & $169(6.4)$ & $76(6.0)$ \\
\hline II & $345(12.0)$ & $135(11.4)$ \\
\hline III & $1193(43.0)$ & $527(44.5)$ \\
\hline IV & 995 (38.7) & $421(38.2)$ \\
\hline \multicolumn{3}{|l|}{ Histological type n (\%) } \\
\hline Sarcoma & $76(4.3)$ & $20(2.8)$ \\
\hline Leiomyosarcoma & $532(28.3)$ & $215(26.6)$ \\
\hline
\end{tabular}




\begin{tabular}{|c|c|c|}
\hline Variable & $\begin{array}{l}\text { Training Cohort } \\
(n=2702)\end{array}$ & $\begin{array}{l}\text { Validation Cohort } \\
(n=1159)\end{array}$ \\
\hline Adenosarcoma & $124(4.4)$ & $45(3.8)$ \\
\hline Stromal sarcoma & $455(14.1)$ & $207(15.5)$ \\
\hline Carcinosarcoma & $1515(48.8)$ & $672(51.3)$ \\
\hline \multicolumn{3}{|l|}{ SEER stage $n(\%)$} \\
\hline Localized & $1116(37.4)$ & $537(42.5)$ \\
\hline Regional & $825(29.1)$ & $321(28.2)$ \\
\hline Distant & $761(33.5)$ & $301(29.3)$ \\
\hline \multicolumn{3}{|l|}{ AJCC stage $n(\%)$} \\
\hline I & $1225(41.0)$ & $573(45.1)$ \\
\hline II & $236(9.7)$ & $84(8.1)$ \\
\hline III & $572(20.0)$ & $222(20.0)$ \\
\hline IV & $669(29.3)$ & $280(26.9)$ \\
\hline \multicolumn{3}{|c|}{ Surgery status n (\%) } \\
\hline Yes & $2516(90.8)$ & $1090(92.4)$ \\
\hline No/Unknown & $186(9.2)$ & $69(7.6)$ \\
\hline \multicolumn{3}{|c|}{ Radiotherapy status n (\%) } \\
\hline Yes & $782(26.3)$ & $313(24.6)$ \\
\hline No/Unknown & $1920(73.7)$ & $846(75.4)$ \\
\hline \multicolumn{3}{|c|}{ Chemotherapy status n (\%) } \\
\hline Yes & $1420(52.4)$ & $598(53.2)$ \\
\hline No/Unknown & $1282(47.6)$ & $561(46.8)$ \\
\hline
\end{tabular}

\section{Variable screening and multivariate Cox regression analysis results}

According to the Cox stepwise regression analysis, tumors' primary site, years of diagnosis and surgery site were excluded for further study due to no difference in the prognosis for US patients. Multivariate Cox regression analyses demonstrated that age at diagnosis [hazard ratio $(H R)=1.0116, p<0.001$ ], being black (HR $=1.1698, p<0.05)$, single (HR $=1.2181$ vs. married, $p<0.05)$, SDW (HR $=1.2965$ vs. married, $p<$ 0.001 ), tumor size ( $>50 \mathrm{~mm}, \mathrm{HR}=1.4861 \mathrm{vs}$. $\leq 50 \mathrm{~mm}, p<0.001$; tumor size unknown, $\mathrm{HR}=1.3345, p<$ 0.01 ), higher pathology grade(Grade III,HR $=7.3773$ vs. pathology grade I, $p<0.001 ; \mathrm{IV}, \mathrm{HR}=7.0185, p<$ 
0.001 ), SEER stage (regional, $\mathrm{HR}=1.8809$ vs. localized, $p<0.001$; distant, $\mathrm{HR}=2.5199, p<0.001$ ), and higher AJCC stage (III,HR $=1.7459$ vs. AJCC stage $\mathrm{I}, p<0.001 ; \mathrm{IV}, \mathrm{HR}=2.2275, p<0.001)$ were all independent risk factors. Meanwhile, we found that leiomyosarcoma ( $\mathrm{HR}=0.6550$ vs. sarcoma, $p<0.01)$, carcinosarcoma ( $H R=0.6099$ vs. sarcoma, $p<0.01$ ), insurance (no insurance $H R=1.4851, p<0.01$ ), receiving surgery therapy (no/unknown $\mathrm{HR}=2.7559, p<0.001$ ), adjuvant radiotherapy (no/unknown $\mathrm{HR}=$ 1.3267, $p<0.001$ ), and adjuvant chemotherapy (no/unknown $\mathrm{HR}=1.5355, p<0.001$ ) were protective factors for surviving US. The results also indicated that other race, other marital status, pathology grade II, adenosarcoma, stromal sarcoma and AJCC stage II were not significant risk factors $(P>0.05)$. The results of multivariate Cox regression analysis were presented Table 2. 
Table 2

Selected variables in the SEER database by multivariate Cox regression analysis (training cohort)

\begin{tabular}{llll} 
Variable & Hazard ratio & $95 \% \mathrm{Cl}$ & $p$ value \\
\hline Age at diagnosis & 1.0116 & $1.0061-1.0172$ & $0.000^{\star * *}$ \\
\hline Race & & & \\
\hline White & Reference & & \\
\hline Black & 1.1698 & $1.0225-1.3383$ & $0.022^{*}$ \\
\hline Other & 0.8481 & $0.6698-1.0739$ & 0.171
\end{tabular}

Marital status

Married Reference

\begin{tabular}{llll} 
Single & 1.2181 & $1.0436-1.4217$ & $0.012^{\star}$ \\
\hline SDW & 1.2965 & $1.1230-1.4969$ & $0.000^{\star \star \star}$ \\
\hline Other & 1.2816 & $0.9740-1.6863$ & 0.076
\end{tabular}

Insurance record

Yes Reference

$\begin{array}{llll}\text { No } & 1.4851 & 1.1367-1.9404 & 0.004^{\star *}\end{array}$

Tumor size

$\leq 50 \mathrm{~mm} \quad$ Reference

$\begin{array}{llll}>50 \mathrm{~mm} & 1.4861 & 1.2674-1.7425 & 0.000 \text { *** }\end{array}$

$\begin{array}{llll}\text { Unknown } & 1.3345 & 1.0802-1.6487 & 0.007 \text { ** }\end{array}$

Pathological grade

\begin{tabular}{llll} 
I & Reference & & \\
II & 1.3135 & $0.7662-2.2518$ & 0.321 \\
\hline III & 7.3773 & $4.5441-11.9771$ & $0.000 * \star *$ \\
IV & 7.0185 & $4.3528-11.3165$ & $0.000 * * *$
\end{tabular}

Histological type

Sarcoma Reference

SDW Separated, divorced, and widowed, SEER Surveillance, Epidemiology, and End Results, HR hazard ratio, AJCC American Joint Committee on cancer

${ }^{\star} p<0.05,{ }^{\star \star} p<0.01,{ }^{* \star *} p<0.001$ 


\begin{tabular}{|c|c|c|c|}
\hline Variable & Hazard ratio & $95 \% \mathrm{Cl}$ & $p$ value \\
\hline Leiomyosarcoma & 0.6550 & $0.4839-0.8866$ & $0.006 * \star$ \\
\hline Adenosarcoma & 0.6288 & $0.3919-1.0089$ & 0.054 \\
\hline Stromal sarcoma & 0.9274 & $0.6623-1.2984$ & 0.661 \\
\hline Carcinosarcoma & 0.6099 & $0.4535-0.8202$ & $0.001 * \star$ \\
\hline \multicolumn{4}{|l|}{ SEER stage } \\
\hline Localized & \multicolumn{3}{|l|}{ Reference } \\
\hline Regional & 1.8809 & $1.3831-2.5580$ & $0.000 * \star \star$ \\
\hline Distant & 2.5199 & $1.6708-3.8005$ & $0.000 * \star \star$ \\
\hline \multicolumn{4}{|l|}{ AJCC stage } \\
\hline I & \multicolumn{3}{|l|}{ Reference } \\
\hline II & 1.0136 & $0.7096-1.4478$ & 0.941 \\
\hline III & 1.7459 & $1.2843-2.3735$ & $0.000 * \star \star$ \\
\hline IV & 2.2275 & $1.4818-3.3484$ & $0.000 \star \star \star$ \\
\hline \multicolumn{4}{|l|}{ Surgery status } \\
\hline Yes & \multicolumn{3}{|l|}{ Reference } \\
\hline No/Unknown & 2.7559 & $2.2503-3.3751$ & $0.000 * * \star$ \\
\hline \multicolumn{4}{|c|}{ Radiotherapy status } \\
\hline Yes & \multicolumn{3}{|l|}{ Reference } \\
\hline No/Unknown & 1.3267 & $1.1567-1.5216$ & $0.000 * \star \star$ \\
\hline \multicolumn{4}{|c|}{ Chemotherapy status } \\
\hline Yes & \multicolumn{3}{|l|}{ Reference } \\
\hline No/Unknown & 1.5355 & $1.3509-1.7453$ & $0.000 \star \star \star$ \\
\hline \multicolumn{4}{|c|}{$\begin{array}{l}\text { SDW Separated, divorced, and widowed, SEER Surveillance, Epidemiology, and End Results, } H R \text { hazar } \\
\text { ratio, AJCC American Joint Committee on cancer }\end{array}$} \\
\hline${ }^{\star} p<0.05,{ }^{* \star} p<0.0$ & & & \\
\hline
\end{tabular}

Nomogram construction 
The nomogram was constructed for predicting the 1-, 3-, and 5-year CSS based on the multivariate Cox regression analysis (Fig. 2). The pathological grade was set as reference scale ranging from 0 to 100 because it had the largest coefficient absolute value. The probabilities of 1-, 3- and 5-year CSS could be easily calculated by adding the points value of each variable. .As shown in Fig. 2, pathological grade contributed the greatest influence to CSS, followed by age at diagnosis, surgery status, SEER stage, AJCC stage, histological grade, chemotherapy, insurance record, tumor size, race, radiotherapy, and finally marital status.

\section{Nomogram comparison and evaluation}

The C-indexes in both training and validation cohorts were higher than the AJCC 7th edition staging system $(0.796$ vs. $0.706,0.767$ vs. 0.713$)$, indicating that our new model showed better discriminative ability. Furthermore, AUCs of the training cohort for the 1-,3-5-year CSS demonstrated superior were significantly larger $(0.842,0.845,0.860$, respectively) than the traditional system $(0.755,0.772$, and 0.774 , respectively). Likewise, the ROC values were also significantly larger $(0.833,0.798,0.797$ at $1-3-, 5$-year vs0.763, 0.741, and 0.747, respectively) (Fig. 3).

\section{Validation and calibration of the nomogram}

The NRI values for the 1-, 3- and 5-year CSS rates in the training cohort were $64.6 \%$ (95\% confidence interval $[\mathrm{Cl}]=55.3-73.5 \%), 59.0 \%(95 \% \mathrm{Cl}=50.7 \%-67.9 \%)$ and $62.2 \%(95 \% \mathrm{Cl}=52.8-71.4 \%)$, respectively, and in the validation cohort, $47.2 \%(95 \% \mathrm{Cl}=25.0-63.1 \%), 37.6 \%(95 \% \mathrm{Cl}=14.1-51.4 \%)$ and $29.9 \%(95 \%$ $\mathrm{Cl}=7.4-55.0 \%$ ), respectively. Moreover, the IDI values for the 1-, 3- and 5-year CSS rates in the training cohort were $8.64,9.63$ and $9.50 \%$, respectively, and $3.98,5.79$ and $5.88 \%$ in the validation cohort (all $p<$ $0.001)$. These results all indicated that the nomogram predicted prognosis with greater accuracy than that of the AJCC model.

Calibration plots which predicting the 1-, 3-and 5-year CSS for the training and validation cohorts were nearly identical to the practical observations, which demonstrated that the new model had great calibration ability (Fig. 4).

\section{Clinical usefulness}

Finally, DCA curve was used to evaluate the clinical usability and benefits of the nomogram. Compared to the AJCC staging system, all the DCA curves showed larger net benefits of the new model in both the training and validation cohorts (Fig. 5). These results demonstrated that the new model had better clinical benefits than the AJCC staging system.

\section{Discussion}

Uterine sarcoma is a group of rare malignant mesenchymal tumors with various histologic types but aggressive progresses, poor prognosis [3]. Up to now, there is no efficient prognostic staging system that could help to estimate CSS at diagnosis in US patients. 
Nomogram is a statistical tool which could provide accurate predictions for malignant tumors by a simple graphical presentation. This convenient nomogram could provide accurate individualized predictions for specified points. Recently it had been developed for several cancers such as NSCLC hepatocellular carcinoma (HCC), and adult skin melanoma $[16,17,18]$. However, few nomograms have been constructed for US patients. Zhou et al identified 6-gene-based prognostic signature for US [19]. Li et al evaluated the benefit of adjuvant radiotherapy for uterine leiomyosarcoma and carcinosarcoma [20]. The latest and largest study done by Mona Hosh et al identified 13089 cases of uterine sarcoma diagnosed from 2000 to 2012 [10]. To our knowledge, this study was the first time to develop a comprehensive prognostic nomogram to predict the 1-, 3-, and 5-year CSS for US based on the SEER database.

Pathological grade, age, surgery, AJCC stage, SEER stage, histological differentiation, chemotherapy, insurance record, tumor size, ethnicity, radiotherapy and marital status were identified as the prognostic factors of the CSS through multivariate Cox regression. Among them, the most notable depressed prognosis for CSS of US patient is pathological grade. It was revealed that the survival rates of patients with grade III and IV were worse compared to those grade I patients, which was consistent with previous studies [21]. However, it had no significant differences between patients of grade III and grade IV.

Age at diagnosis played the secondary crucial role in our model, although the exact mechanism remained unclear. Mona Hosh et al also found that the incidence of US increased with increasing age, while aged 50 years or older patients had worse survival than those younger patients [10]. Several reports have shown that the elder patients did not derive the same benefit from cancer treatments as the general population in clinical trials, which might because of the declines of organ function. The present study indicated that black US patient, tumor size $(>5.0 \mathrm{~cm})$ and histological type were all associated with poor prognosis for patients with US. Other studies also indicated that progression and poor survival rates were more common in patients with black, carcinosarcoma and larger tumors [22].

More interestingly, we discovered the marital status influenced CSS of Uterine Sarcoma for the first time. Evidences showed that unmarried patients exhibited shorter OS and CSS compared with married patients in lung and liver cancer [24, 23]. In the present study, patients of Separated, Divorced and Widowed (SDW) had the worst survival compared to those married patients, followed by the single patients. This might because that marriage and a spouse could not only provide financial support, but also relieve the depression and anxiety for the patients that caused by cancer [25]. Insurance was also strongly associated with the prognosis of uterine sarcoma. Patients with insurance could receive better medical support, less economical and less psychological distress compared with uninsured patients. These new information could therefore further help clinicians to make more effective clinical decisions.

In addition, we also found that surgery status, SEER stage, AJCC stage and radiotherapy status would affect the survival probability for US. Surgery is the gold standard treatment for US [2]. In addition, among these clinical parameters, the surgery status had the highest discriminating power in our study. Another 
important factor was the localized stage of US at initial diagnosis. The patients that had distant metastatic have more aggressive disease than whose only had localized disease.

Radiation therapy is usually performed in advanced uterine sarcoma patients. Several retrospective researches suggested radiotherapy after surgery could decrease pelvic recurrence, but not for distant metastases [26]. In contrast, Wong et al found that adjuvant pelvic radiotherapy might improve OS and reduce local recurrence for leiomyosarcoma [27]. In our study, Fig. 2 clearly showed both surgery and radiotherapy could improve the survival on the 1-, 3-, and 5-year CSS probabilities in US patients.

Notably, we identified chemotherapy provides patients with a better prognosis for the first time. There are very few studies focusing on chemotherapy and patient prognosis for US patients in SEER database. Efficacious chemotherapy to achieve prolonged survival in those with both early and advanced-stage US patients has been elusive. Hensley et al evaluated the role of 4 cycles of gemcitabine and docetaxel in 25 high-grade uterine leiomyosarcoma patients, and found that prolonged PFS and OS than before [28]. However, Littell et al compared gemcitabine-docetaxel with observation in 110 stage I uLMS patients after surgery, and found no significance difference in disease-free or OS or recurrence in two groups [29]. Our nomogram showed that chemotherapy had an even higher discriminating power than radiotherapy. This data on chemotherapy could help clinicians to choose individualized adjuvant treatment after surgery.

NRI, IDI, DCA, discrimination and calibration were used to evaluate the performance of the nomogram in order to compare with TNM-based AJCC staging. The survival nomogram performed better discrimination with C-indexes of $0.796,0.767$ for the training and validation cohort, as the values only 0.706 and 0.713 for the AJCC stage. As shown in Fig. 3, all the 1-, 3-, and 5-year AUC values of the AJCC staging system were significantly lower than those of the nomogram. The plots resembling 45-degree lines indicated the predictions of our nomogram were well calibrated. Furthermore, the NRI and IDI both demonstrated that the new nomogram improved the predictive ability than the AJCC staging system. At last, DCA curves were used to assess the clinical effectiveness of the nomogram. Our results showed that the 1-, 3-, and 5year DCA curves for CSS exhibits better clinical effectiveness for predicting survival compared to the traditional AJCC staging system in both training and validation cohorts.

This study, of course, still had several limitations. First, adjuvant hormonal therapy was not included in this nomogram, which might be due to the hormonal therapy was not routinely recommended as postoperative treatment in all histological types of US. Second, SEER database did not use the FIGO staging system for US patients, instead the SEER stage and AJCC stage were used. Third, some potential predictive variable such as serum marker, neutrophil-to-lymphocyte ratio were not included in this study because of these data' absence in the SEER database. Forth, our study excluded the patients diagnosed after 2015. The NCCN guideline of Uterine Neoplasms modified the pathology types of uterine sarcoma since 2016. More recently diagnosed patients and patients of several rare pathological types were excluded to make sure sufficient follow-up so that we could adequately assess the association of treatment with survival. 


\section{Conclusions}

In summary, we have established a novel nomogram to predict the 1-, 3-, and 5-year CSS for US based on the SEER database. Our nomogram could be used as a valuable and effective tool to help clinicians to provide more individualized treatment and individualized survival prediction in clinical practice.

\section{Abbreviations}

US: Uterine sarcoma; CSS: Cancer-specific survival; AJCC: American Joint Committee on Cancer; SEER: Surveillance, Epidemiology, and End Results database; SDW: Separated, Divorced and Widowed; C-index: Concordance index; AUC: Area under the curve; NRI: Net reclassification improvement; IDI: Integrated discrimination improvement; DCA: Decision-curve analysis; HR: Hazard ratio. ICD-0-3: International Classification of Diseases for oncology, Third Edition morphology code.

\section{Declarations}

\section{Acknowledgements}

We would like to thank the SEER program for providing open access to the database.

\section{Conflict of Interest}

All authors declare that they have no conflict of interests.

\section{Funding}

The study was supported by the General projects of Key research and Development program in Natural Foundation of Shaanxi Province, China (Grant No.2017SF-015; 2019SF-139).

\section{Authors' contributions}

Yuan-jie Li and Jun Lyu contributed equally to the work.

Yuan-jie Li and Jun Lyu analyzed the data and performed the conceptualization and formal analysis. Chen Li performed statistical analysis and data interpretation. Hai-rong He and Jin-feng Wang were responsible for the quality control of data and data extraction. Yue-ling Wang contributed to the writingreview and editing. Jing Fang performed investigation, literature research. Jing Ji designed the study and submitting manuscript. All authors contributed to writing of the manuscript and approved the final version.

\section{Competing interests}

All authors declare no competing interests. 


\section{Ethics approval and consent to participate}

No applicable. This data used in this study were from public database (SEER database). Approval was waived by the local ethics committee, as SEER data is publicly available and de-identified.

\section{Consent for publication}

No applicable.

\section{Availability of data and materials}

Data from the SEER program is available for public. The data supporting the conclusions of this article are available in the Surveillance Epidemiology, and End Results (SEER) database (https://seer.cancer.gov/).

\section{References}

1. Mbatani N, Olawaiye AB., Prat J. Uterine sarcomas. Int J Gynaecol Obstet. 2018;143(Suppl.2): 51-58. https://doi.org/10.1002/ijgo.12613

2. Trope CG., Abeler VM., Kristensen GB. Diagnosis and treatment of sarcoma of the uterus. A review. Acta Oncol. 2012;51(6): 694-705. https://doi.org/10.3109/0284186X.2012.689111.

3. Rizzo A, Pantaleo MA, Saponara M, Nannini M. Current status of the adjuvant therapy in uterine sarcoma: A literature review. World J Clin Cases. 2019;7(14): 1753-1763. https://doi.org/10.12998/wjcc.v7.i14.1753

4. Kristen N Ganjoo, Uterine sarcomas. Curr Probl Cancer. 2019;43(4): 283-288. https://doi.org/10.1016/j.currproblcancer.2019.06.001.

5. Wu TI, Chang TC, Hsueh S, Hsu KH, Chou HH, Huang HJ, et al., Prognostic factors and impact of adjuvant chemotherapy for uterine leiomyosarcoma. Gynecol Oncol. 2006;100(1): 166-172. https://doi.org/10.1016/j.ygyno.2005.08.010

6. Kapp DS, Shin JY, Chan JK. Prognostic factors and survival in 1396 patients with uterine leiomyosarcomas: emphasis on impact of lymphadenectomy and oophorectomy, Cancer 2008;112(4): 820-830. https://doi.org/10.1002/cncr.23245

7. Amin MB, Greene FL, Edge SB, Compton CC, Gershenwald JE, Brookland RK, et al. The Eighth Edition AJCC Cancer staging Manual: Continuing to build a bridge from a population-based to a more "personalized" approach to cancer staging, CA Cancer J Clin. 2017; 67(2): 93-99. https://doi.org/10.3322/caac.21388

8. Nipp R, Tramontano AC, Kong CY, Pandharipande P, Dowling EC, Schrag D, et al., Disparities in cancer outcomes across age, sex, and race/ethnicity among patients with pancreatic cancer. Cancer Med. 2018; 7(2):525-535. https://doi.org/10.1002/cam4.1277 
9. Doepker MP, Holt SD, Durkin MW, Chu CH, Nottingham JM, Triple-Negative Breast Cancer: A Comparison of Race and Survival, Am Surg. 2018; 84(6): 881-888.

10. Hosh M, Antar S, Nazzal A, Warda M, Gibreel A, Refky B. Uterine sarcoma: analysis of 13,089 cases based on surveillance, epidemiology, and end results database, Int J Gynecol Cancer. 2016;26(6): 1098-104. https:// dx.doi.org/10.1097/IGC.0000000000000720

11. Surveillance Research Program, National Cancer Institute SEER*Stat software (seer.cancer.gov/seerstat) version <SEER*Stat 8.3.6.1>.

12. National Cancer Institute. Surveillance, Epidemiology, and End Results Program. Available at http://seer.cancer.gov. 2020; Assessed July 14.

13. Yang J, Li YJ, Liu QQ, Li L, Feng AZ, Wang TY, et al. Brief introduction of medical database and data mining technology in big data era. Journal of Evidence-Based Medicine. 2020; 13(1): 57-69. https://doi.org/10.1111/jebm.12373

14. Steyerberg EW, Vickers AJ, Cook NR, Gerds T, Gonen M Obuchowski, N, et al. Assessing the performance of prediction modelsm epidemiology: A Framework for Traditional and Novel Measures. Epidemiology 2010;21(1):128-138. https://doi: 10.1097/EDE.0b013e3181c30fb2

15. Vickers AJ, Elkin EB. Decision curve analysis: a novel method for evaluating prediction models, Med Decis Making. 2006; 26(6): 565-74. https://doi.org/10.1177/0272989X06295361

16. Grimes DA. The nomogram epidemic: resurgence of a medical relic, Ann Intern Med. 2008;149(4):273-5. DOI: 10.7326/0003-4819-149-4-200808190-00010

17. Balachandran VP, Gonen M, Smith JJ, DeMatteo RP. Nomograms in oncology: more than meets the eye, Lancet Oncol. 2015;16(4) : 173-80. https://doi.org/10.1016/S1470-2045(14)71116-7

18. Chen Y, Liao F, Cao L.Web-based nomograms for predicting the prognosis of adolescent and young adult skin melanoma, a large population-based real-world analysis. Transl Cancer Res, 2020;9(11): 7103-7112. https://dx.doi.org/10.21037/tcr-20-1295

19. Zhou JG, Zhao HT, JIN SH, Tian X, Ma H. Identification of a RNA-seq-based signature to improve prognostics for uterine sarcoma, Gynecol Oncol. 2019;155(3): 499-50719. https://doi.org/10.1016/j.ygyno.2019.08.033

20. Li Y, Ren HT, Wang JM. Outcome of adjuvant radiotherapy after total hysterectomy in patients with uterine leiomyosarcoma or carcinosarcoma: a SEER-based study, BMC Cancer. 2019;19(1): 697. https://doi.org/10.1186/s12885-019-5879-7

21. Sait HK, Anfinan NM, Sayed ME. El, AlkhayyatSS, Ghanem AT, Abayazid RM, et al. Uterine sarcoma: Clinico-pathological characteristics and outcome. Saudi Med J. 2014; 35(10): 1215-1222.

22. Brooks SE, Zhan M, Cote T, Baquet CR. Surveillance, epidemiology, and end results analysis of 2677 cases of uterine sarcoma 1989-1999. Gynecol Oncol. 2004;93(1): 204-208. https://doi.org/10.1016/j.ygyno.2003.12.029

23. Varlotto JM, McKie K, Voland RP, Flickinger JC, DeCamp MM, Maddox D, et al. The role of race and economic characteristics in the presentation and survival of patients with surgically resected nonsmall cell lung cancer. Front Oncol. 2018;8: 146. https://doi.org/10.3389/fonc.2018.00146. 
24. Wu Y, Ai Z, Xu G. Marital status and survival in patients with non-small cell lung cancer: An analysis of 70006 patients in the SEER database. Oncotarget., 2017;8(61):103518-103534. https://doi.org/10.18632/oncotarget.21568.

25. Goldzweig G, Andritsch E, Hubert A, Brenner B, Walach N, Perry S, et al. Psychological distress among male patients and male spouses: what do oncologists need to know? Ann Oncol., 2010; 21(4): 877883. https://doi.org/10.1093/annonc/mdp398.

26. Champetier C, Hannoun-Levi JM, Resbeut M, Azria D, Salem N, Tessier E, et al. Postoperative radiotherapy of uterine sarcoma: a multicentric retrospective study. Cancer Radiother. 2011; 15(2): 89-96. https://doi.org/10.1016/j.canrad.2010.05.005.

27. Wong P, Han K, Sykes J, Catton C, Laframboise S, Fyles A, et al. Postoperative radiotherapy improves local control and survival in patients with uterine leiomyosarcoma. Radiat Oncol. 2013;24(8): 128. https://doi.org/10.1186/1748-717X-8-128.

28. Hensley ML, Ishill N, Soslow R, Larkin J, Abu-Rustum N, Sabbatini P, et al. Adjuvant gemcitabine plus docetaxel for completely resected stages I-IV high grade uterine leiomyosarcoma: Results of a prospective study. Gynecol Oncol., 2009; 112(3): 563-7. https://doi.org/10.1016/j.ygyno.2008.11.027

29. Littell RD, Tucker LY, Raine-Bennett T, Palen TE, Zaritsky E, Neugebauer R, et al. Adjuvant gemcitabinedocetaxel chemotherapy for stage I uterine leiomyosarcoma: Trends and survival outcomes, Gynecol Oncol., 2017;147(1): 11-17. https://doi.org/10.1016/j.ygyno.2017.07.122

\section{Figures}




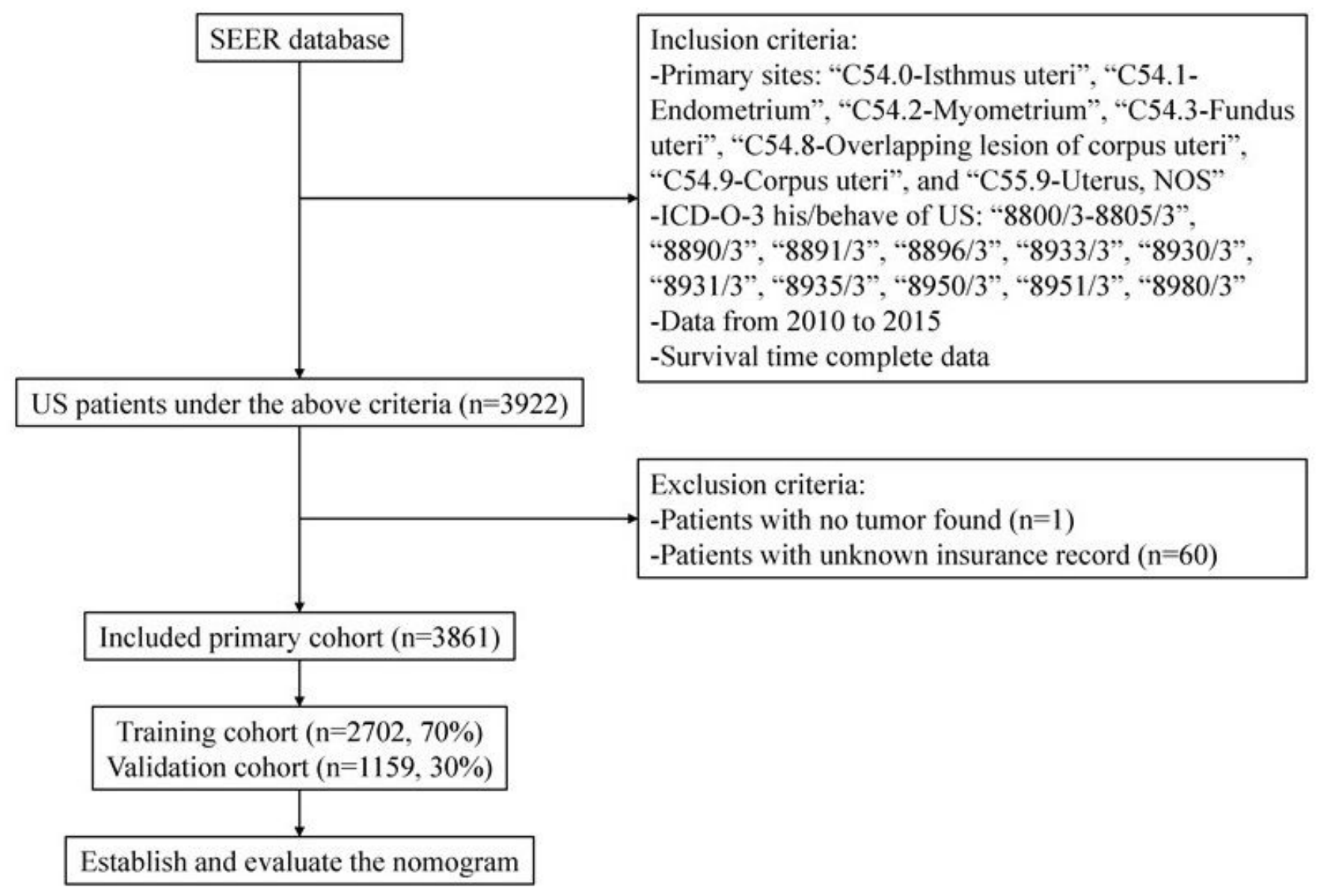

Figure 1

Research flowchart 
Points

Age

Race

Marital

Insurance

Tumorsize

Grade

Hist

Stage

AJCC

Surgery

Radiation

Chemotherapy

Total Points

1-years Survival Probability

3-years Survival Probability

5-years Survival Probability

\begin{tabular}{|c|c|c|c|c|c|c|c|c|c|c|}
\hline 0 & 10 & 20 & 30 & 40 & 50 & 60 & 70 & 80 & 90 & 100 \\
\hline
\end{tabular}
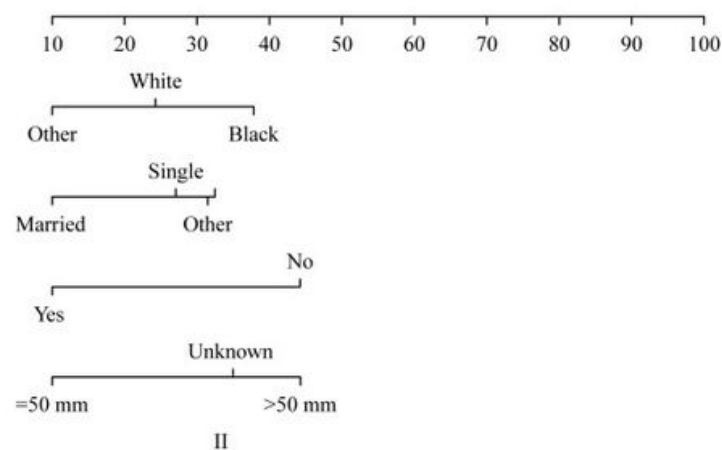

II

$$
\text { I }
$$
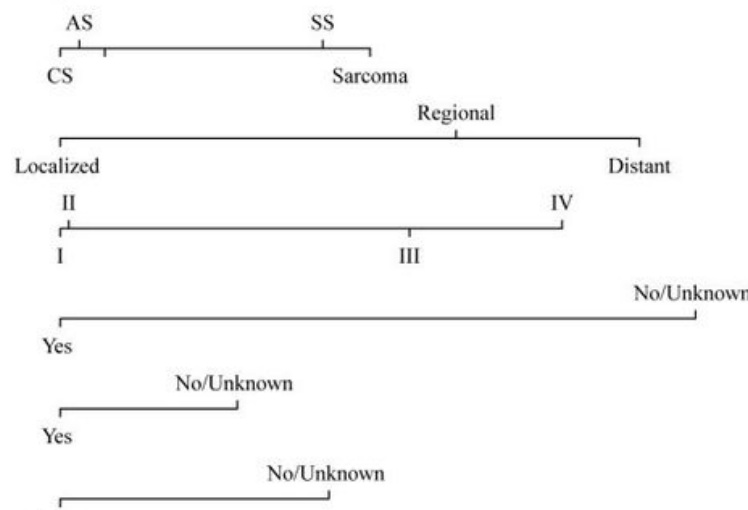

Yes
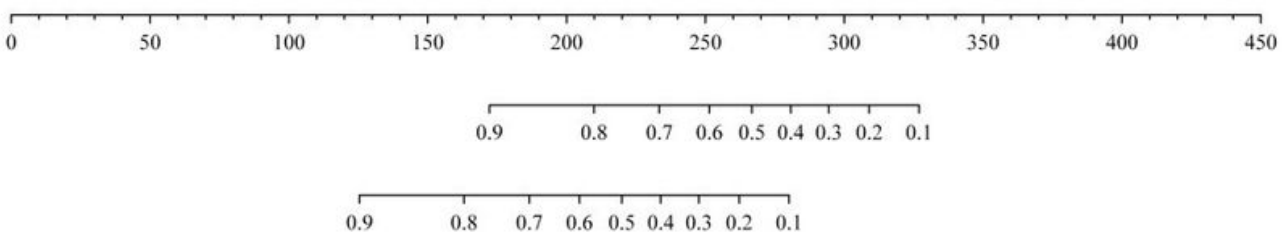

\begin{tabular}{lllllllllll}
\hline & 0.9 & 1 & 0.8 & 0.7 & 0.6 & 0.5 & 0.4 & 0.3 & 0.2 & 0.1
\end{tabular}

Figure 2

Nomogram predicting 1-, 3- and 5-year survival. (AJCC, 7th AJCC tumor stage) 

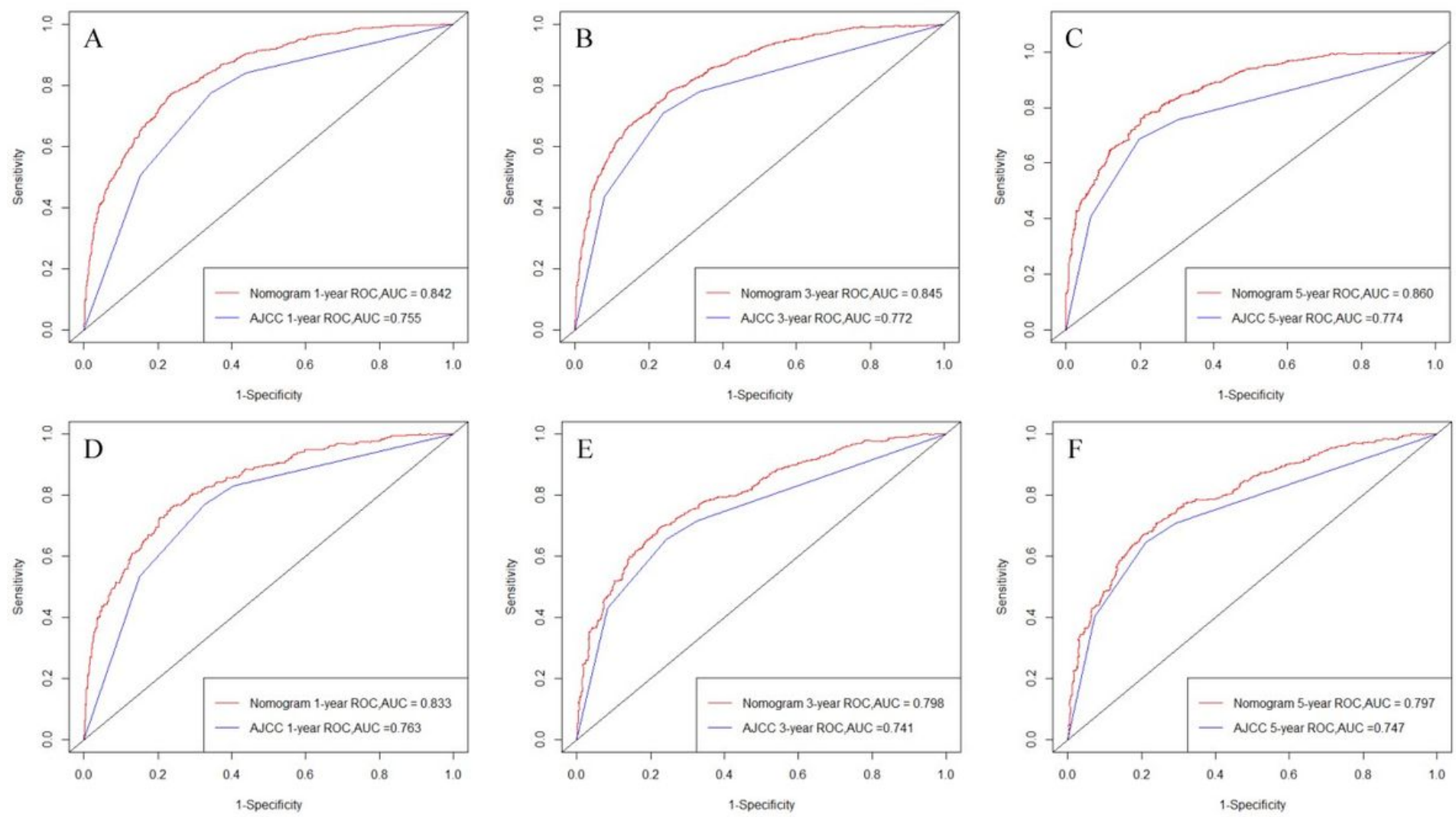

\section{Figure 3}

ROC curves. ROC curve analyses were generated to test the performance evaluating between the new model and the traditional AJCC model, by the AUC. A, B and C came from the training set, $D, E$, and F came from the validation set. 

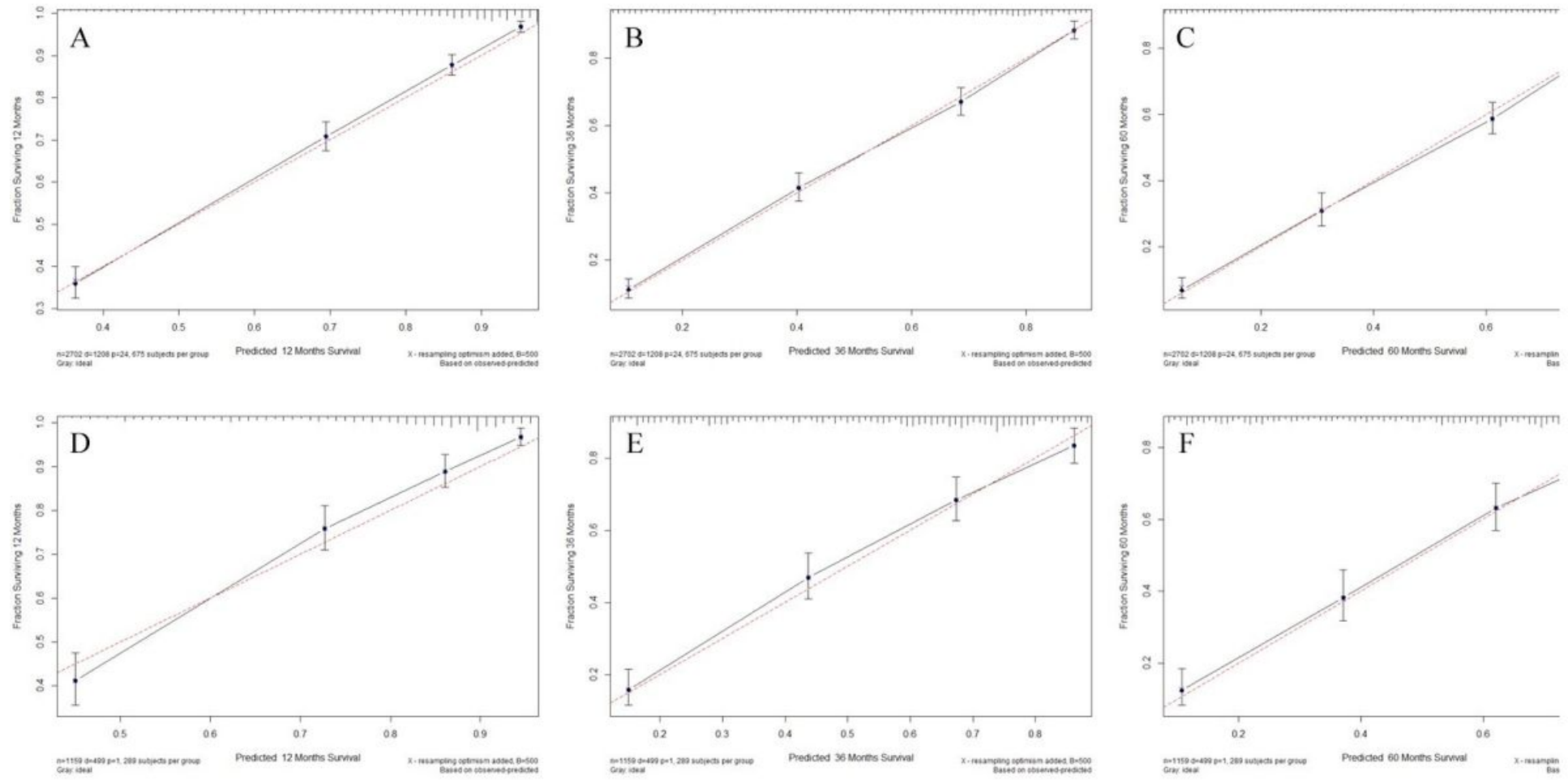

Figure 4

Calibration curves. Calibration curves for 1-, 3- and 5-year CSS depict the calibration of each model in terms of the agreement between the predicted probabilities and observed outcomes of the training cohort $(A, C, E)$ and validation cohort $(B, D, F)$. 

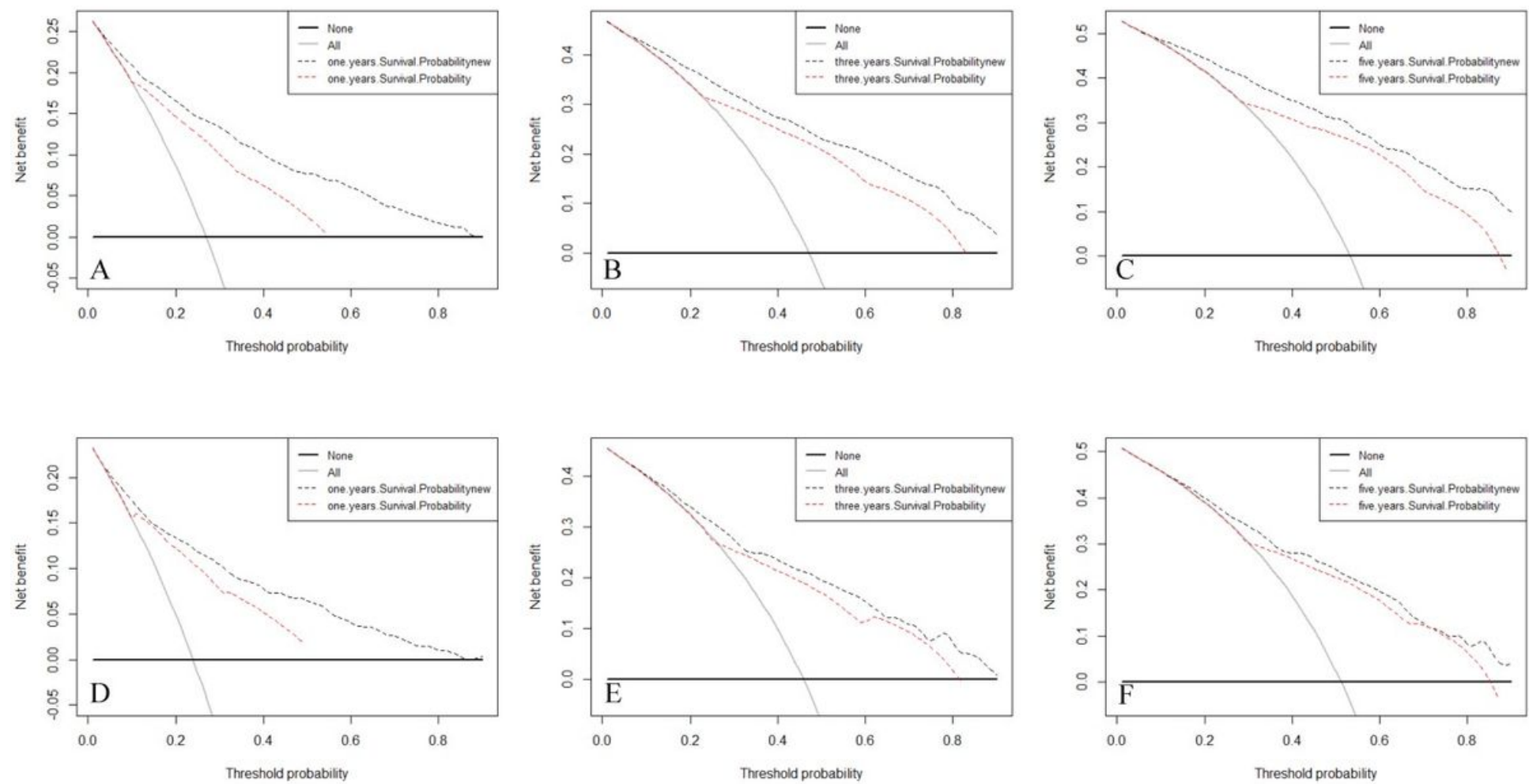

Figure 5

Decision curve analysis curves. Decision curve analysis of the training $(A, B, C)$ and validation cohorts $(\mathrm{D}, \mathrm{E}, \mathrm{F})$ 